\title{
Prospects of Gravitational Wave Detections from Common Envelope Evolution with LISA
}

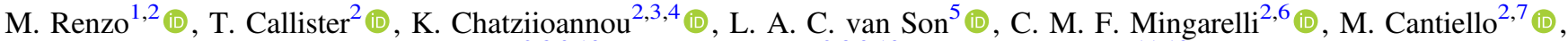 \\ K. E. S. Ford ${ }^{2,8,9,10}$ (D) B. McKernan ${ }^{2,8,9,10}$ (iD), and G. Ashton ${ }^{11,12}$ (D) \\ ${ }^{1}$ Department of Physics, Columbia University, New York, NY 10027, USA; mrenzo@ flatironinstitute.org \\ ${ }^{2}$ Center for Computational Astrophysics, Flatiron Institute, 162 5th Ave, New York, NY 10010, USA \\ ${ }^{3}$ Department of Physics, California Institute of Technology, Pasadena, California 91125, USA \\ ${ }^{4}$ LIGO Laboratory, California Institute of Technology, Pasadena, CA 91125, USA \\ ${ }^{5}$ Center for Astrophysics - Harvard \& Smithsonian, 60 Garden Street, Cambridge, MA 02138, USA \\ ${ }^{6}$ Department of Physics, University of Connecticut, 196 Auditorium Road, U-3046, Storrs, CT 06269-3046, USA \\ ${ }^{7}$ Department of Astrophysical Sciences, Princeton University, Princeton, NJ 08544, USA \\ ${ }^{8}$ Department of Science, Borough of Manhattan Community College, City University of New York, New York, NY 10007 USA \\ ${ }^{9}$ Department of Astrophysics, American Museum of Natural History, New York, NY 10024 USA \\ ${ }^{10}$ Physics Program, CUNY Graduate Center, City University of New York, New York, NY 10016 USA \\ ${ }_{11}$ Royal Holloway University of London, Egham, Surrey, TW20 0EX, UK \\ ${ }^{12}$ School of Physics and Astronomy, Monash University, VIC 3800, Australia \\ Received 2021 January 28; revised 2021 June 30; accepted 2021 July 1; published 2021 October 4
}

\begin{abstract}
Understanding common envelope (CE) evolution is an outstanding problem in binary evolution. Although the CE phase is not driven by gravitational wave (GW) emission, the inspiraling binary emits GWs that passively trace CE dynamics. Detecting this GW signal would provide direct insight into gas-driven physics. Even a non-detection might offer invaluable constraints. We investigate the prospects of detection of a Galactic CE by LISA. While the dynamical phase of the CE is likely sufficiently loud for detection, it is short and thus rare. We focus instead on the self-regulated phase that proceeds on a thermal timescale. Based on population-synthesis calculations and the (unknown) signal duration in the LISA band, we expect $\sim 0.1-100$ sources in the Galaxy during the mission duration. We map the GW observable parameter space of frequency $f_{\mathrm{GW}}$ and its derivative $\dot{f}_{\mathrm{GW}}$, remaining agnostic on the specifics of the inspiral and find that signals with signal-to-noise ratios $>10$ are possible if the CE stalls at separations such that $f_{\mathrm{GW}} \gtrsim 2 \times 10^{-3} \mathrm{~Hz}$. We investigate the possibility of misidentifying the signal with other known sources. If the second derivative $\ddot{f}_{\mathrm{GW}}$ can also be measured, the signal can be distinguished from other sources using a GW braking index. Alternatively, coupling LISA with electromagnetic observations of peculiar red giant stars and/or infrared and optical transients, might allow for the disentangling of a Galactic CE from other Galactic and extragalactic GW sources.
\end{abstract}

Unified Astronomy Thesaurus concepts: Common envelope binary stars (2156); Common envelope evolution (2154); Gravitational wave astronomy (675); Binary stars (154); Interacting binary stars (801)

\section{Introduction}

Common envelope (CE) evolution is one of the most challenging processes to study in the evolution of binary stars. CE evolution was originally proposed by Paczyński (1976) to account for the short orbital separations observed in cataclysmic variables. Despite its importance for many categories of stellar objects, the details of this process remain elusive.

A CE is typically assumed to occur when the evolution of a binary system leads to dynamically unstable mass transfer, causing the accretor to be engulfed in the donor's envelope. This results in a CE binary, in which the core of the donor star and the accretor star coexist within a shared envelope. Drag forces then drive the inspiral of the binary, injecting orbital energy (and angular momentum; Nelemans et al. 2000) into the $\mathrm{CE}$. Depending on the complex coupling between the orbit and the envelope structure, the $\mathrm{CE}$ can ultimately be ejected, or, alternatively, the two stars can merge. We expand on the current understanding of CE evolution in Section 1.1.

Evolution through a CE phase is a key step in the formation of almost any compact binary system from an isolated binary, including cataclysmic variables (e.g., Paczyński 1976), subdwarf B stars (e.g., Han et al. 2003; Igoshev et al. 2020) and stripped stars (Götberg et al. 2020), double white dwarfs (WD, e.g., Nandez et al. 2015), X-ray binaries (e.g., Podsiadlowski et al. 2002; Chen et al. 2020a), binary neutron stars (e.g., Tauris et al. 2017; Kruckow et al. 2018; Vigna-Gómez et al. 2020), black hole neutron-star binaries (e.g., Kruckow et al. 2018; Chattopadhyay et al. 2020; Broekgaarden et al. 2021), black hole WD binaries (e.g., Sberna et al. 2020), and binary black holes (e.g., Belczynski et al. 2016; Kruckow et al. 2018). CE evolution also produces the tightest binaries which can in turn make the fastest runaway stars from isolated systems if disrupted by the explosion of one star (e.g., Justham et al. 2009; Renzo et al. 2019; Evans et al. 2020; Neunteufel 2020). Finally, CE preceeds the majority of stellar mergers, ${ }^{13}$ which are common across the entire mass range of stars (e.g., de Mink et al. 2013; Zapartas et al. 2017; Temmink et al. 2020) and may possibly lead to the formation of Thorne-Zytkow objects (e.g., Thorne \& Żytkow 1977).

The final phases of a CE-the envelope ejection or the merger event-can produce visible electromagnetic (EM) transients called luminous red novae (LRN). The detection of the pre-transient period decay of V1309Sco (Tylenda et al. 2011) unambiguously

\footnotetext{
${ }^{13}$ We neglect here the possibility of (close to) head-on collisions between stars in dense stellar environments which can also lead to mergers (e.g., Glebbeek et al. 2009; Di Carlo et al. 2020; Renzo et al. 2020).
} 
connected this type of transient to CE. LRNe exhibit a variety of morphologies (e.g., Pastorello et al. 2020), possibly related to the variety of $\mathrm{CE}$ progenitors and outcomes possible.

Numerical simulations of $\mathrm{CE}$ evolution remain challenging (e.g., Ricker \& Taam 2008; Ohlmann et al. 2016; MacLeod et al. 2018; Chamandy et al. 2020; Cruz-Osorio \& Rezzolla 2020; Sand et al. 2020, see also Ivanova et al. 2013b, 2020 for reviews). The main difficulties relate to the large range of temporal and spatial scales that need to be resolved in $\mathrm{CE}$ evolution. Moreover, there are several possible energy sources other than the orbit that need to be considered during $\mathrm{CE}$ evolution, such as recombination energy (e.g., Ivanova et al. 2013a; Ivanova 2018; Sand et al. 2020), accretion energy (e.g., Voss \& Tauris 2003; MacLeod \& Ramirez-Ruiz 2014), and energy released by nuclear burning (e.g., Ivanova et al. 2002). Both where the energy is released, and how it is transported through the $\mathrm{CE}$ also have a crucial role in determining the outcome (e.g., Sand et al. 2020; Wilson \& Nordhaus 2020). Therefore, CE evolution remains one of the major uncertainties in the modeling of binary evolution and stellar populations.

The inspiral within a $\mathrm{CE}$ is not driven by gravitational wave (GW) emission. Nevertheless, the binary inside of a CE, consisting of the core of the donor and the companion star, has a time-varying mass quadrupole moment and hence radiates GWs. If detectable, GWs from the binary inside of a CE will passively trace the gas-drag-driven inspiral and could provide invaluable insight into the CE process. Conversely, because of the low density of the envelope of giant stars (which is possibly further lowered by the energy injection during the CE), any GW signal from the envelope itself is unlikely to be detectable.

The GWs radiating from a Galactic CE binary would be in the $\mathrm{mHz}$ band and thus could be detectable by space-based GW detectors, such as the Laser Interferometer Space Antenna (LISA, Baker et al. 2019), and TianQin (Huang et al. 2020). This can be expected because of the existence of double-WD verification binaries for LISA (e.g., Stroeer \& Vecchio 2006), which likely went through at least one CE phase (e.g., Korol et al. 2017). LISA is currently scheduled for launch in 2034 and will commence operations in the $\mathrm{mHz} \mathrm{GW}$ frequency range shortly thereafter. Its projected mission lifetime is $5-10 \mathrm{yr}$, during which time it is expected to detect a wide variety of GW signals in the $\mathrm{mHz}$ band, from supermassive black hole binary mergers to Galactic compact binaries with a fraction of a solar mass.

Here, we examine the prospects for observing the inspiral of two Galactic stellar cores within a CE with LISA. Such signals are not accessible to ground-based detectors (e.g., LIGO-Aasi et al. 2015, Virgo-Acernese et al. 2015, and KAGRASomiya 2012) as they are restricted to frequencies above a few to tens of $\mathrm{Hz}$. At these frequencies, CE binaries are expected to either have merged or have ejected the envelope. In fact, Ginat et al. (2020) showed that LISA could detect the portion of the $\mathrm{CE}$ inspiral proceeding on a dynamical timescale ( $\sim$ days).

However, not all CE events are expected to rapidly complete their evolution over a dynamical timescale (Ivanova et al. 2013b). A significant fraction of the envelope may be ejected on thermal timescales much longer than the dynamical timescale (e.g., Meyer \& Meyer-Hofmeister 1979; Glanz \& Perets 2018; Michaely \& Perets 2019). Currently, the duration and occurrence rate of this so-called "self-regulated inspiral" is very uncertain (e.g., Meyer \& Meyer-Hofmeister 1979; Fragos et al. 2019; Sand et al. 2020; Igoshev et al. 2020), but if it is of the order of years or longer, this phase is likely to constitute the most promising CE target for LISA.

Constraints placed by low-frequency GWs on CE evolution will be from direct detection/non-detection. These constraints will be complementary to those obtained by ground-based detectors which observe the aftermath of successful CE events in massive binaries where the envelope has been ejected and the binary merged under the influence of GWs. The properties and rate of such detections can be used to place constraints on the CE properties, for example, CE efficiency (e.g., Wong et al. 2021; Zevin et al. 2020). Space-based detectors, on the other hand, offer the possibility of studying binaries while they undergo $\mathrm{CE}$, possibly including systems that might fail to eject the envelope. Provided the signal can be distinguished from other potential $\mathrm{mHz} \mathrm{GW}$ sources, space-based detectors will give us direct insight into gas-driven dynamics.

In particular, the $\mathrm{GW}$ frequency of the signal $\left(f_{\mathrm{GW}}\right)$ can constrain the orbital separation at which the self-regulated inspiral stalls and the frequency change $\left(\dot{f}_{\mathrm{GW}}\right)$ and event duration can constrain the rapidity of the inspiral and thus the internal gas density of the CE (and potentially its radial distribution). Even a non-detection would offer invaluable constraints on the occurrence rate, the orbital separation during the self-regulated inspiral, and the duration of this phase, which are not accessible by any other means.

\subsection{Phases of Common envelope Evolution}

Because of the aforementioned complexity of $\mathrm{CE}$ evolution and the diversity of binary systems that can evolve through this phase (both in terms of masses and evolutionary stages at the onset of the $\mathrm{CE}$ ), a complete and exhaustive picture of this phenomenon does not yet exist. Nevertheless, several phases that might subsequently occur during the $\mathrm{CE}$ have been identified (e.g., Podsiadlowski 2001; Ivanova et al. 2013b; Fragos et al. 2019; Ivanova et al. 2020) and are outlined in Figure 1. Below we emphasize which are potentially interesting targets for GW observations. It is possible that not every binary evolving through a CE will experience all of these phases (Ivanova et al. 2013b; Law-Smith et al. 2020).

a. Onset of the common envelope-Mass transfer through a Roche lobe overflow (RLOF) can become dynamically unstable and result in the initiation of a CE for several reasons:

1. an increasing rate of overflow, either because the Roche lobe shrinks or because it expands slower than the donor's star radius;

2. because the mass transfer timescale is much shorter than the accretor thermal timescale, the accretor is pushed out of thermal equilibrium, which leads to its radial expansion until the accretor too fills its Roche lobe (e.g., Nariai \& Sugimoto 1976);

3. because of Darwin instability that redistributes angular momentum between the stellar spins and the orbit (Darwin 1879), causing the orbit to shrink;

4. because of mass loss from the $L_{2}$ Lagrangian point, causing large orbital energy loss, which accelerates the evolution to proceed on a dynamical timescale (e.g., Pejcha et al. 2017).

These processes can cause the accretor to enter the envelope of the donor, starting the $\mathrm{CE}$ on a timescale that is either thermal or dynamical. Possible pre-CE mass ejection can also occur, depending on what drives the $\mathrm{CE}$ initiation 


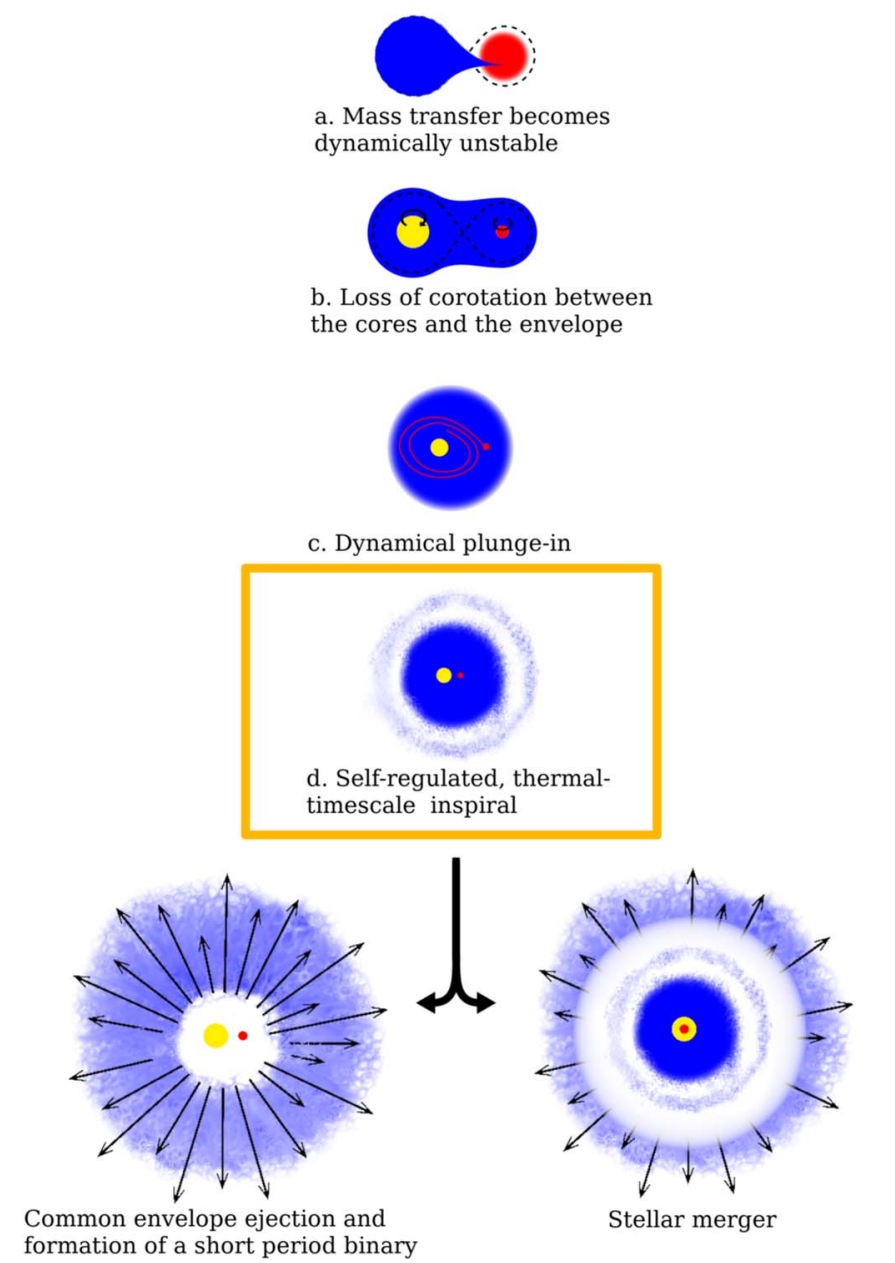

Figure 1. Schematic representaion of all possible phases during a common envelope event. We focus on the most promising source of GW in the LISA band, i.e., the thermal-timescale self-regulated phase at step d. However, dynamical phases such as the plunge-in at step c or the final ejection or merger might also be detectable (e.g., Ginat et al. 2020).

(e.g., Pejcha et al. 2017; MacLeod et al. 2017; MacLeod \& Loeb 2020). When RLOF becomes dynamically unstable, the separation, $a$, between the core of the donor and the accretor is still wide and of the order of the Roche radius of the donor $a \gtrsim R_{\mathrm{RL}, 1}$. Except for binaries already involving two compact objects (e.g., AM CVn systems, Liu et al. 2021), this is likely to correspond to a $\mathrm{GW}$ signal of frequency $f_{\mathrm{GW}}$ too low for the LISA band.

b. Loss of co-rotation-Soon after the initiation of the CE, the envelope will rapidly stop co-rotating with the binary (even under the optimistic assumption that the envelope was initially co-rotating with the donor's core). The timescale for the loss of co-rotation depends on how the CE was initiated at step (a), but it varies between the tidal timescale up to a thermal timescale (e.g., Podsiadlowski 2001; Fragos et al. 2019). The loss of corotation still happens at separations wider than could be detectable in the LISA band, but it allows for the onset of strong gas drag which drives subsequent $\mathrm{CE}$ evolution.

c. Dynamical plunge-in-After the loss of co-rotation, with the envelope density still only slightly perturbed (Fragos et al. 2019), a phase of dynamical plunge-in happens. This rapid inspiral can take (a fraction of) an orbital period and possibly decrease the orbital separation $a$ to zero (i.e., a prompt merger). Alternatively, it can lead to a prompt ejection of the CE (e.g.,
Law-Smith et al. 2020). However, this is not always the case. The orbital energy liberated in this very fast plunge-in could expand the $\mathrm{CE}$, leading to a decrease in gas drag and thus stalling the inspiral, setting up the conditions for phase $(d)$ (see below). At which separation $a_{\text {post-plunge }}$ the plunge-in stall is not likely to be universal and is not yet fully understood. Podsiadlowski (2001) suggested that $a_{\text {post-plunge }}$ corresponds to the location where the orbital energy equates the envelope binding energy, however, De Marco et al. (2011) caution about the subtleties of how the binding energy is defined. Depending on the pre- and post-plunge-in separations, this dynamical phase could be detectable in GWs for Galactic binaries (e.g., Ginat et al. 2020). However, because of its short duration ( $\sim$ hours-days), it is not the most promising source (see Section 4). Should one happen within the Galaxy during the LISA mission, it would produce a frequency change $\dot{f}_{\mathrm{GW}}$ much larger than expected from the GW emission and possibly be associated with a detectable EM transient. The plunge-in phase can also produce an eccentric binary within the CE (Ivanova et al. 2013b; Sand et al. 2020) with potential implications for the GW signal they might produce.

d. Self-regulated inspiral-Assuming the plunge-in did not produce a prompt merger, what follows is a relatively slow phase of self-regulated inspiral, that can last several thermal timescales (10-10 5 yr, e.g., Meyer \& Meyer-Hofmeister 1979; Clayton et al. 2017; Fragos et al. 2019; Chamandy et al. 2020; Igoshev et al. 2020). During the self-regulated inspiral, the quasi-Keplerian motion of the binary slowed by gas drag progressively injects orbital energy and angular momentum into the $\mathrm{CE}$. Other energy sources can intervene and radiative transport becomes relevant during this CE phase (e.g., hydrogen recombination can occur if it happens in a sufficiently optically thick layer; Fragos et al. 2019). Depending on the separation $a_{\text {post-plunge }}$ (and the masses of the binary), this phase might be the most promising for detecting a GW signal since it could allow for a slowly ${ }^{14}$ varying $f_{\mathrm{GW}}$ that can be integrated over several years to build up signal-to-noise ratio (SNR). However, we emphasize that this self-regulated inspiral might not happen for all $\mathrm{CE}$ events (see, for example, the short timescale for subdwarf B-type progenitors inferred by Igoshev et al. 2020, or the numerical results for neutron-star progenitors by Law-Smith et al. 2020).

e. CE ejection or merger-Finally, the $\mathrm{CE}$ will end with one last dynamical phase during which either the envelope is ejected (possibly because of the intervention of energy sources other than the orbit) or one of the stars of the binary inside the $\mathrm{CE}$ fills the equipotential surface distinguishing it from the other (improperly, its Roche lobe) leading to the final merger (e.g., Podsiadlowski 2001; Ivanova et al. 2002). In the cases where the CE is successfully ejected, the surviving binary is left with a final $a_{\text {post-CE }}$ orbital separation smaller than the initial radius of the donor. What governs the binary post-CE orbital evolution depends on the specific details of the system (magnetic braking, tidal evolution, wind mass loss, or possibly GW emission). The short duration of phase (c) makes it an unpromising target for GW searches. However, it would result in a large $\dot{f}_{\mathrm{GW}}$ for a brief moment and likely be accompanied by a detectable EM transient.

In the rest of this study, we will focus on potential signals from a long-lived, self-regulated inspiral (step $d$ ) unless

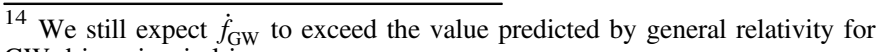
$\mathrm{GW}$-driven inspiral in a vacuum.
} 


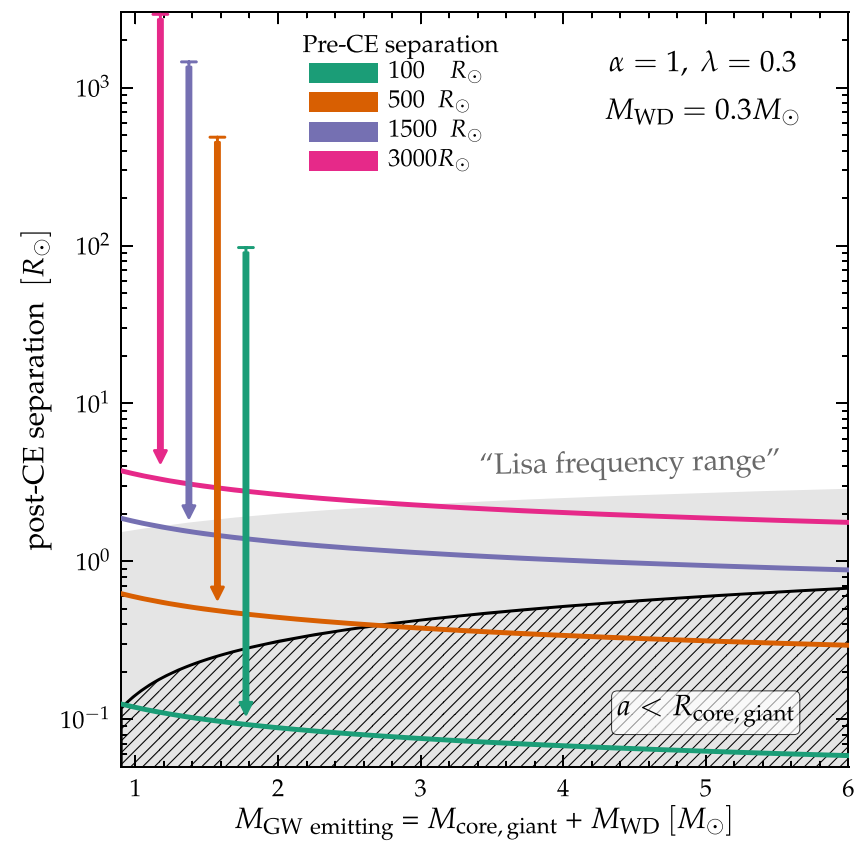

Figure 2. Estimates of the post-CE separations using the $\alpha \lambda$ energy formalism. Given a pre-CE separation (different colors in plot) and the GWs emitting mass ( $x$ axis), the post-CE separation can be determined. We show a range of GW-emitting masses, consisting of the core mass of the donor star plus the mass of the companion WD $\left(0.3 M_{\odot}\right.$ in this plot). We assume $\alpha=1$ and $\lambda=0.3$ for illustrative purposes. The gray band shows where the corresponding $\mathrm{GW}$ signal approximately would be in the LISA frequency range $\left(10^{-4} \mathrm{~Hz} \lesssim f_{\mathrm{GW}} \lesssim 0.1 \mathrm{~Hz}\right)$. Systems with post-CE separations within or below this band therefore potentially experience a phase during which their GW emission could be detectable by LISA. The hatched area corresponds to post-CE separation smaller than the core radius, which is presumably merging systems.

otherwise stated. In Section 2 we show that CE binaries of various mass are expected to cross or end within the LISA GW frequency band. We then consider the issue of whether a typical binary crossing the LISA band can be detected in Section 3 and use population synthesis simulations to estimate the number of sources expected at any given time in the Galaxy in Section 4. Section 5 deals with the possible "stealth bias," that is, the possibility of misinterpreting the detection of GWs from a $\mathrm{CE}$ as another kind of GW source, and we consider how EM observation can help resolve this bias in Section 6. We further discuss our results in Section 7 before concluding in Section 8 .

\section{Common Envelope Evolution across the LISA Band}

For a CE event to cross the LISA frequency band and produce a detectable GW signal, the mass of the binary inside the CE needs to be sufficiently large. This favors systems where both stars are evolved and have dense and well defined cores (or are only what is left of their original core, like WDs). On top of this, the separation of the binary inside the $\mathrm{CE}$ needs to correspond to $\mathrm{GW}$ frequencies roughly $10^{-4} \mathrm{~Hz} \lesssim f_{\mathrm{GW}} \lesssim 0.1 \mathrm{~Hz}$ (gray band in Figure 2 ), bound at the low end by the population of unresolved Galactic WD and at the high end by the instrument response function (e.g., Robson et al. 2019). The existence of verification WD binaries in the LISA band, which are likely to have formed through a CE event, suggests that CE binaries crossing the LISA band may exist.

To illustrate that some binaries will indeed cross the LISA band during a CE, we use a population synthesis approach (see also Section 4). In rapid population synthesis calculations, CE is typically treated as an instantaneous event. This is usually a sufficient approximation since the duration of a $\mathrm{CE}$ is at most of order of the thermal timescale (depending on the mass, luminosity, and radius of the $\mathrm{CE}$, this ranges from several hundreds to several thousand years), which is much shorter than the entire evolution of a binary system. To relate the pre$\mathrm{CE}$ initial conditions to the estimated post-CE configuration, conservation of energy is usually used ( $\alpha \lambda$-algorithms, see e.g., Webbink 1984; De Marco et al. 2011), although algorithms considering conservation of angular momentum also exist (e.g., Nelemans et al. 2000).

Figure 2 shows the post-CE separation $\left(a_{\text {post-CE }}\right)$ as a function of the mass that would be contributing to the GW emission ( $M_{\mathrm{GW} \text { emitting }}$ ). In a rapid population synthesis calculation, each binary system would drop instantaneously from its pre-CE separation to the post-CE separation (as indicated by the vertical arrows). For illustrative purposes, we assume fully efficient use of the orbital energy to eject the envelope and no extra energy source (i.e., $\alpha=1$ ) and $\lambda=0.3$ as a typical value for the binding energy parameter of giant donors (e.g., De Marco et al. 2011). Increasing either value shifts the curves to larger post-CE separations.

In Figure 2 we assume $M_{\mathrm{GW}}$ emitting to be the (helium) core mass of the donor star at the end of the main sequence plus $0.3 M_{\odot}$ assumed to be the mass of a WD companion. We chose this value to reflect the highest rate of initiation of Galactic $\mathrm{CE}$ events from the simulations described in Section 4, which is expected for systems with $M_{\text {core }} \simeq 0.5 M_{\odot}$ and $M_{\mathrm{WD}} \simeq 0.3 M_{\odot}$. Although the combination of core masses with this particular WD mass might not be realistic throughout the range shown, we have also performed the same calculation assuming a more massive companion of $1.4 M_{\odot}$ (mimicking a Chandrasekhar-mass WD or a neutron star). The resulting curves are shown in Figure 6 in Appendix $\mathrm{C}$ where each curve moves up to larger post-CE separations, but most curves still intersect the LISA band (gray area). For large core radii, the final dynamical phase (e.g., in Figure 1 above) might start at frequencies in the LISA band (hatched area in Figure 2). The systems ending at separation smaller than the He core radii could result in mergers rather than successful common envelope ejections. Whether this leads to a detectable GW signal or not requires further investigation.

We use the solar metallicity models from Pols et al. (1998) to obtain the (helium) core mass of the donor from their total masses. Our core masses are a lower bound since we neglect core growth due to the ashes of (hydrogen) shell burning. The gray shaded area indicates core separations corresponding to a GW signal of frequency $10^{-4} \mathrm{~Hz} \lesssim f_{\mathrm{GW}} \lesssim 0.1 \mathrm{~Hz}$, roughly within the LISA band. Binaries crossing this gray area or ending within it (e.g., verification WD binaries) are potential candidates for GW detection of the CE evolution with LISA depending on the duration of the $\mathrm{CE}$ event. The duration cannot be obtained from a population synthesis approach; it either needs to be modeled with challenging multi-dimensional hydrodynamic simulations or, as we will argue below, it might be directly constrained with observation of the GW signal.

We return to a full population synthesis calculation of the rate of events in Section 4 after discussing the observability of GW from a CE in Section 3.

\section{Detectability of Common Envelope in LISA}

We now explore in what regimes we expect LISA to successfully detect $\mathrm{GW}$ signals from binaries undergoing $\mathrm{CE}$. 


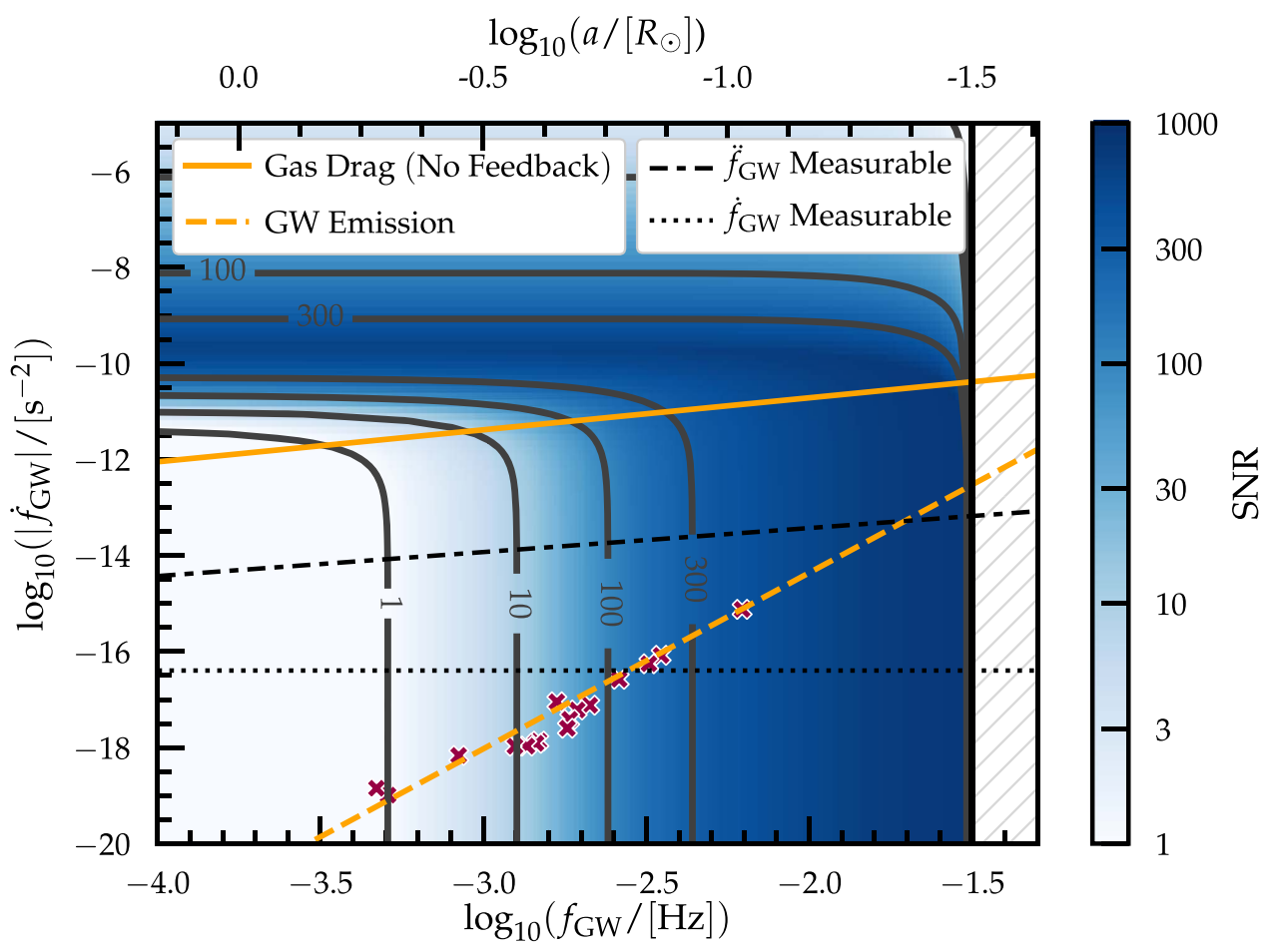

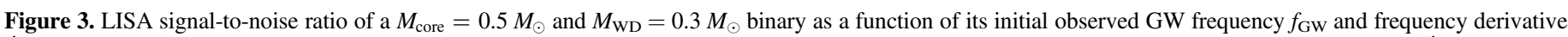

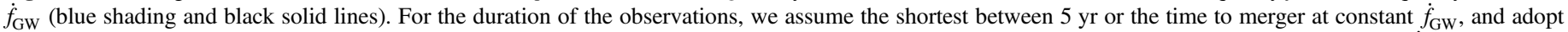

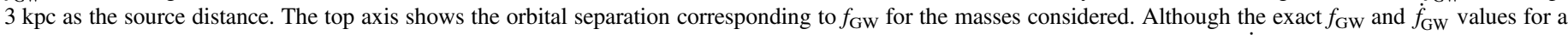

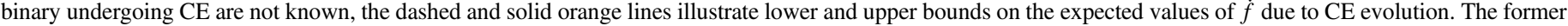

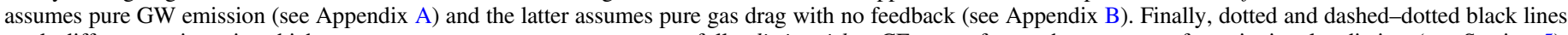

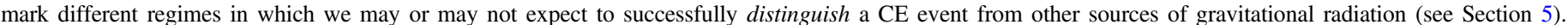

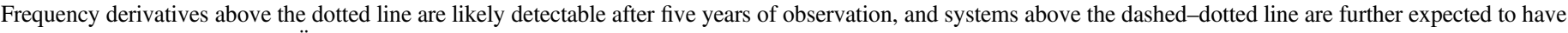

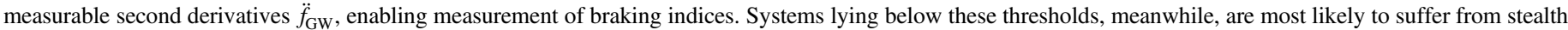
bias or misidentification. The red crosses indicate known verification WD binary.

Since the details of CE are not yet fully understood, we do not know the precise frequency evolution of a GW source undergoing CE. Instead, in Figure 3 we agnostically quantify the expected SNRs with which LISA will observe a $M_{\mathrm{GW} \text { emitting }}=0.5+0.3 M_{\odot}$ binary with an initial GW frequency $f_{\mathrm{GW}}$ and frequency derivatives $\dot{f}_{\mathrm{GW}}$ at a distance of $3 \mathrm{kpc}$. We chose a value for the typical mass of the donor star core $\left(0.5 M_{\odot}\right)$ and the WD companion $\left(0.3 M_{\odot}\right)$ from our population synthesis (see Section 4). Expected SNRs have been averaged over possible sky locations and binary inclinations. Throughout, we assume the standard quadrupole radiation such that $f_{\mathrm{GW}}=2 f_{\text {orb }}$, where $f_{\text {orb }}$ is the (quasi-) Keplerian orbital frequency of the CE binary. We further assume the observation to persist for five years $^{15}$ or until the given binary's frequency reaches $f_{\mathrm{GW}}=10^{-1.5} \mathrm{~Hz}$. Beyond this frequency, the He core would fill its Roche lobe within the CE, initiating the dynamical phase that leads to a prompt merger (bottom right-hand side of Figure 1) and termination of the GW signal. Additional details of the SNR calculation are given in Appendix A.

Although the true rate $\dot{f}_{\mathrm{GW}}$ at which a binary's GW frequency evolves under $\mathrm{CE}$ is not known, we can nevertheless place sensible bounds on frequency evolution of a CE binary. In Figure 3, the orange dashed line shows the frequency derivative $\dot{f}_{\mathrm{GW}}\left(f_{\mathrm{GW}}\right)$ due solely to the emission of GWs. For a given total mass, this is a strict lower bound on the evolution rate of a binary undergoing CE. Meanwhile, the orange solid line shows the value of $\dot{f}_{\mathrm{GW}}\left(f_{\mathrm{GW}}\right)$ expected in the case of pure gas drag, neglecting the reaction of the envelope and assuming $\rho=10^{-6} \mathrm{~g} \mathrm{~cm}^{-3}$,

$\overline{15}$ The SNR is proportional to the square root of the observing time.
$T=9 \times 10^{5} \mathrm{~K}$ to represent the density and temperature deep inside the shared CE (see Appendix B). This provides a strict upper limit since in nature we expect the envelope to expand due to the energy injected into the envelope by the inspiral, lowering the gas drag on the binary inside the CE. Generally, we expect CE binaries to lie between these two bounds. Under these constraints, we see that systems occupying a large portion of the remaining $f_{\mathrm{GW}}-\dot{f}_{\mathrm{GW}}$ phase space are detectable with LISA. In particular, systems whose gravitational wave frequency is $f_{\mathrm{GW}} \gtrsim 10^{-3} \mathrm{~Hz}$ (corresponding to orbital separations less than $\sim 0.3 R_{\odot}$ for our fiducial masses) yield SNR $\gtrsim 10$.

Although galactic binaries undergoing self-regulated $\mathrm{CE}$ evolution may be detectable by LISA, they are not necessarily identifiable as such. Without additional information or careful consideration, we run the risk of misidentifying such a system as a purely GW-driven compact binary, incorrectly attributing an anomalously high $\dot{f}_{\mathrm{GW}}$ to large component masses rather than accelerated evolution due to CE. This issue of identification will be discussed further in Section 5 below.

\section{Estimate of the Number of Galactic Common Envelope Binaries}

To determine the number of $\mathrm{CE}$ binaries in the Galaxy that LISA (and future GW observatories) might be able to detect, we use the population synthesis code COSMIC $^{16}$ version 3.2

\footnotetext{
${ }^{16}$ https://cosmic-popsynth.github.io/. Our input files and results are available at https://zenodo.org/record/4490011.
} 

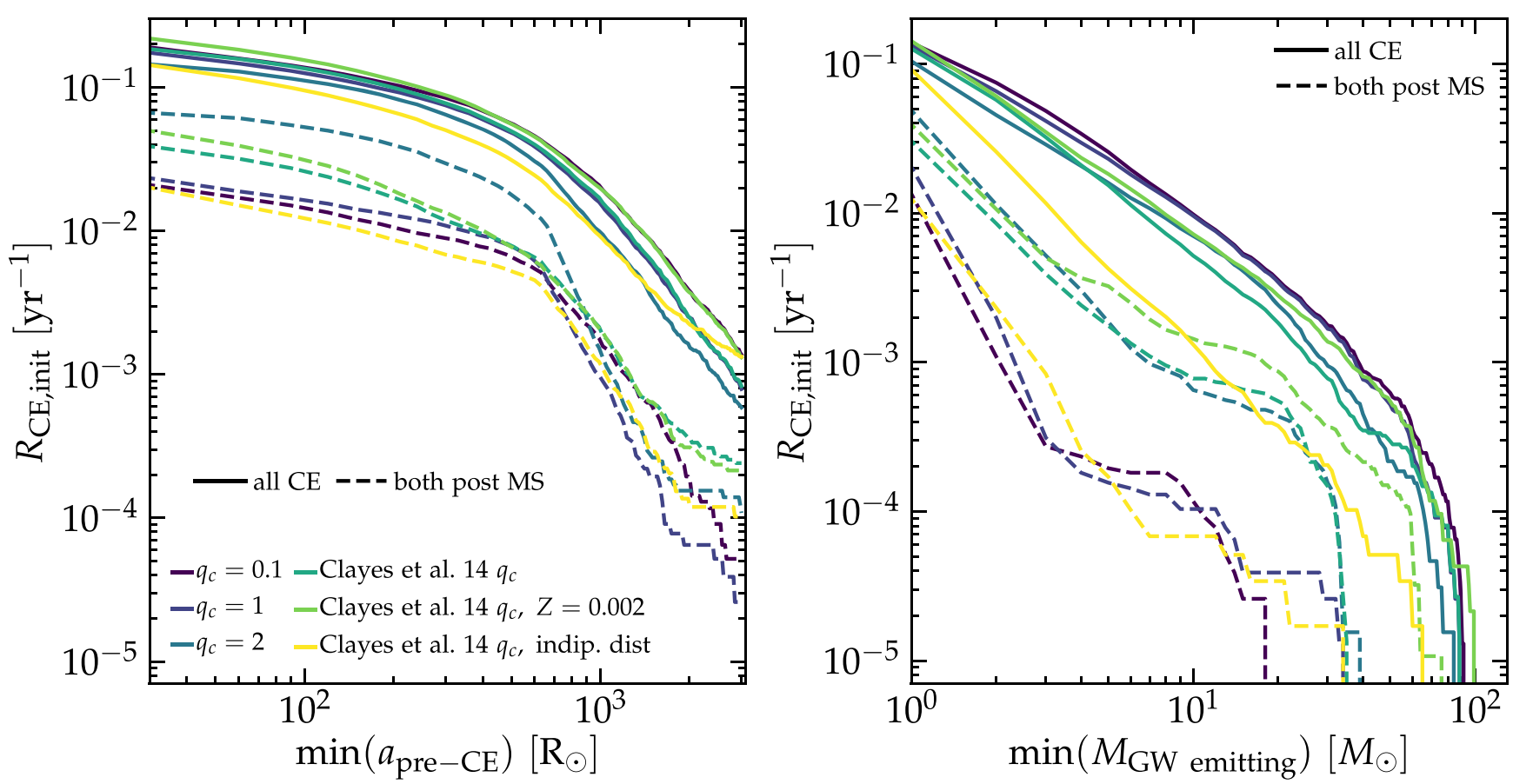

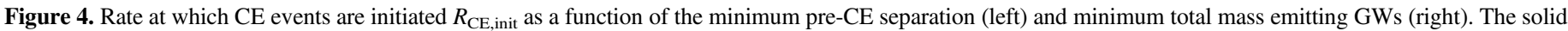

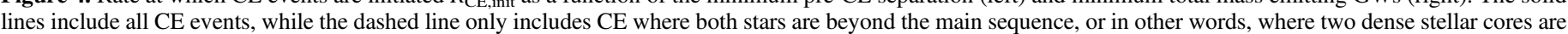
involved. Different colors correspond to different assumptions on the stability of mass transfer ( $q_{c}$, see also text) and initial distribution of binaries (yellow).

(Breivik et al. 2020). As in all rapid binary population synthesis codes, COSMIC treats CE as an instantaneous event, therefore, we obtain from COSMIC the rate at which CE events are initiated $\left(R_{\mathrm{CE} \text {,init }}\right)$ by scaling the number of binaries initiating a $\mathrm{CE}$ with the total mass of the population and multiplying by a star formation rate (SFR) of $3.5 M_{\odot} \mathrm{yr}^{-1}$ (commonly used for Milky-way equivalent galaxies in population synthesis calculations of GW sources, e.g., Dominik et al. 2012). The value of $R_{\mathrm{CE} \text {,init }}$ can be rescaled for an arbitrary SFR, which anyway does not constitute the dominant uncertainty in the determination of the number of Galactic sources.

Population synthesis calculations assume a CE event occurs if one of the following conditions is met: (i) both stars simultaneously fill their Roche lobe (e.g., for initially equal mass ratio systems), (ii) one of the stellar radii exceeds the periastron point-for eccentric binaries, or (iii) when the mass transfer is deemed to be unstable. Case (iii) is most common. Mass transfer stability is decided in COSMIC using critical mass ratio values $q_{c}$ (Breivik et al. 2020, see also Hurley et al. 2002; Pavlovskii et al. 2017; Vigna-Gómez et al. 2020). Whenever the mass ratio between the donor star and the accretor is larger than a given threshold $M_{\text {donor }} / M_{\text {accretor }} \geqslant q_{c}$, mass transfer is defined to be unstable and a $C E$ is initiated. Different $q_{c}$ values can be used for each evolutionary phase of the donor star to reflect the changes in thermal timescales as stars evolve.

In our fiducial run-labeled as "Clayes et al. $14 q_{c}$ " in Figure 4, we assume the donor-type dependent $q_{c}$ values ${ }^{17}$ from Table 2 of Claeys et al. (2014), a metallicity $Z=0.02$, and the correlated initial distributions in primary mass, mass ratio, period, and eccentricity from Moe \& Di Stefano (2017). We explore several variations of our fiducial run, keeping the same setup except one of the following:

${ }^{17}$ However, Claeys et al. (2014) define the critical mass ratio as $M_{\text {accretor }} / M_{\text {donor }}$.
1. constant $q_{c}$ for all evolutionary phases of the donor starwe consider values from $q_{c}=0.1$ (more likely to enter $\mathrm{CE}$ ) to $q_{c}=2$ (less likely to enter $\mathrm{CE}$ );

2. lower metallicity $Z=0.002$;

3. independent and uncorrelated initial distributions with a Kroupa et al. (1993) initial mass function for the primary stars, a flat mass-ratio distribution, a log-normal period distribution (e.g., Duchêne \& Kraus 2013), and a thermal eccentricity distribution.

COSMIC is a Monte Carlo population synthesis code (Breivik et al. 2020) and for each population described above, we stop drawing systems when the sampling uncertainty in the initial distributions is less than $10^{-5}$, that is, drawing more systems has an effect smaller than this threshold. More specifically, we check the initial primary and secondary mass distributions in the range $[0,80] M_{\odot}$ and initial orbital period below 1000 days. The number of systems simulated to reach this threshold ultimately determines the total mass of the simulated population, which we use to determine $R_{\mathrm{CE} \text {,init }}$.

These parameter variations do not exhaust the entire range of uncertainties. For example, the fraction of mass that is accreted during stable mass transfer can influence the orbital evolution of the binary and thus modify the chances of a subsequent reverse CE from the initially less massive star to the initially more massive star (e.g., Zapartas et al. 2017). However, we expect the impact of other parameters to be smaller than the impact of varying $q_{c}$ and the initial distributions for binaries.

We find a rate of $\mathrm{CE}$ initiation regardless of the masses involved of $R_{\mathrm{CE} \text {,init }}=0.18_{-0.09}^{+0.02} \mathrm{yr}^{-1}$ for all CE events, and $0.06_{-0.02}^{+0.03} \mathrm{yr}^{-1}$ when considering only CE between two evolved stars (see below). The uncertainties on $R_{\mathrm{CE} \text {,init }}$ are significantly smaller than the uncertainty on the duration of the CE (see Section 1.1). 
Figure 4 shows the rate of $\mathrm{CE}$ initiation that we obtain as a function of the minimum pre-CE separation (left) and the minimum mass emitting GWs (right), i.e., the rate of events with pre-CE separation (or GW-emitting mass) indicated by the abscissa or larger. For example, for our fiducial simulation, about $1.6 \times 10^{-2} \mathrm{CE}$ events are initiated per year with a pre-CE separation $a_{\text {pre-CE }} \geqslant 10^{3} R_{\odot}$. The solid lines in Figure 4 show all $\mathrm{CE}$ events initiated, while the dashed lines focus on $\mathrm{CE}$ events where both stars are evolved beyond the main sequence, meaning both have a well defined, dense core. The latter (dashed lines) represent systems that are more likely to be detectable in GWs.

When considering all CE events, higher $q_{\text {crit }}$ corresponds to lower $R_{\mathrm{CE}}$, init . Conversely, $\mathrm{CE}$ with both stars post-MS, i.e., with a (semi-) degenerate core, might not be the first mass transfer episode in a given binary and their occurrence and rate depend also on what is assumed for previous mass transfer phases. The $\mathrm{CE}$ initiation rate is rather flat for pre-CE separations as large as $a_{\text {pre-CE }} \lesssim 700 R_{\odot}$, while it drops very quickly with increasing $M_{\mathrm{GW}}$ emitting because of the initial mass function.

To determine the mass emitting GWs in each binary, we assume the following: if a star (donor or accretor) is on the main sequence (MS), that is, it lacks a well defined core, we use its total mass in the mass emitting GWs. If a star has evolved beyond the MS, only the core mass adds to the GW-emitting mass. If a star has become a compact object (WD, neutron star, or black hole) again, the total mass of the object adds to the GW-emitting mass. There are two edge cases where our assumptions might overestimate the amount of mass involved in GW emission as the total mass of the binary: CE between two MS stars, and between two compact stars (e.g., a doubledegenerate SNIa progenitor; Dan et al. 2011). However, this has very little effect on our conclusions. With our initial period distribution, CE between two MS stars is relatively rare and they are unlikely to be the most interesting target for LISA, since the lack of dense cores limits the mass involved in the generation of GWs and thus the signal amplitude. Setting instead the total mass emitting GWs to zero for these systems has a negligible effect on the right panel of Figure 4. CE between two compact stars do not occur with our fiducial setup.

Assuming the stellar population of the Galaxy is in equilibrium, an estimate of the current number of Galactic $\mathrm{CE}$ can then be obtained with

$$
N_{\mathrm{CE}}=R_{\mathrm{CE}, \text { init }} \times \Delta t_{\mathrm{CE}},
$$

where $\Delta t_{\mathrm{CE}}$ is the typical duration of a CE event, which is not known. Moreover, what matters for GW detection is not the actual duration $\Delta t_{\mathrm{CE}}$, but the fraction of it spent at a separation corresponding to a $\mathrm{GW}$ frequency in the detector bandpass.

Depending on the evolutionary stage of the CE binary and the model assumed, estimates of the $\mathrm{CE}$ duration $\Delta t_{\mathrm{CE}}$ can range from a dynamical timescale ( days, e.g., Ginat et al. 2020; LawSmith et al. 2020) to a thermal timescale $\left(\sim 10^{5} \mathrm{yr}\right)$, although the radial expansion and luminosity increase of the envelope during the $\mathrm{CE}$ are likely to reduce the relevant thermal timescale to $10^{3}-10^{4}$ yr (e.g., Meyer \& Meyer-Hofmeister 1979; Clayton et al. 2017; Igoshev et al. 2020). Recent hydrodynamical simulations suggest a 10 yr duration (e.g., Fragos et al. 2019; Chamandy et al. 2020).

Based on these previous estimates, assuming a short duration of the $\mathrm{CE}$ of $\sim$ years, the rate of $\mathrm{CE}$ at the low-mass end
$\left(R_{\mathrm{CE} \text {,init }} \approx 0.1 \mathrm{yr}^{-1}\right)$ is sufficiently large that at least one source might be detectable during a $10 \mathrm{yr}$ LISA mission. Considering only $\mathrm{CE}$ with evolved stars (dashed lines), $R_{\mathrm{CE} \text {,init }}$ drops to $\sim 0.5 \times 10^{-2} \mathrm{yr}$, making the expected number of sources only marginally non-zero.

If the self-regulated phase of the $\mathrm{CE}$ instead lasts much longer than the planned LISA mission, there might be upwards of hundreds of detectable sources in the Galaxy $\left(R_{\mathrm{CE} \text {,init }} \approx 0.1\right.$ $\left.\mathrm{yr}^{-1} \times 10^{4} \mathrm{yr} \simeq 1000\right)$. Thus, under the most pessimistic assumptions, there might be only a slim chance of detecting a CE event through LISA, while under more optimistic, but not extreme, assumptions there could be a significant number of target sources available. We return to the possible implications of a non-detection in Section 7.2.

\section{Stealth Bias}

There is an ever growing catalog of potential systems that LISA might be sensitive to, both galactic and extragalactic. Examples include exoplanets (e.g., Tamanini \& Danielski 2019), WD binaries in various stages of their evolution (e.g., Kupfer et al. 2020; Liu et al. 2021), and binary black holes (BBH) in a vacuum or embedded in gas (e.g., Chen et al. 2020b). Our study suggests that CE events are also potentially detectable by LISA, raising the question: how can we distinguish GWs from a $\mathrm{CE}$ from these other possibly confounding sources? In this section we discuss GW-based ways that could be used to conclude that a detected signal is a CE event and the expected bias if we fail to do so. We discuss further EM-based ways of resolving this stealth bias in Section 6.

Depending on the exact frequency evolution of the CE event, there may exist an observational degeneracy between the $\mathrm{CE}$ inspiral and purely GW-driven binaries of higher mass hosting black holes and/or neutron stars. In standard GW analysis that assumes a binary in vacuum is described by general relativity (GR), a measurement of a binary's $f_{\mathrm{GW}}$ and $\dot{f}_{\mathrm{GW}}$ can be used to estimate its mass and distance. If a particular binary is actually undergoing $\mathrm{CE}$, the assumption of vacuum breaks down and the resulting estimates will be biased compared to their true values (see also Chen et al. 2020b for an extended discussion).

This bias is quantified in Figure 5, which shows the chirp mass

$$
M_{c}=\frac{\left(M_{1} M_{2}\right)^{3 / 5}}{\left(M_{1}+M_{2}\right)^{1 / 5}},
$$

(color in top) and distance ( $D$, color in bottom) that we would incorrectly assign to a $0.5+0.3 M_{\odot}$ binary undergoing $\mathrm{CE}$ at $3 \mathrm{kpc}$, if we have measured only its gravitational wave frequency and frequency derivative (and the signal amplitude). We note that for our example binary, the correct value of the chirp mass would be $M_{c}=0.34 M_{\odot}$ (pink line in the top panel). Depending on the exact values of $\left(f_{\mathrm{GW}}, \dot{f}_{\mathrm{GW}}\right)$, we find the possibility of large biases in the inferred parameters, some of them being consistent with BBHs at cosmological distances.

We identify three possible means of breaking this degeneracy between $\mathrm{BBHs}$ and $\mathrm{CE}$ events:

1. Increased observation time could result in a measurement of $\ddot{f}_{\mathrm{GW}}$ (and higher derivatives) in addition to $f_{\mathrm{GW}}$ and $\dot{f}_{\mathrm{GW}}$, enabling a measurement of the binary's braking index. This scenario is further discussed in Section 5.1. 

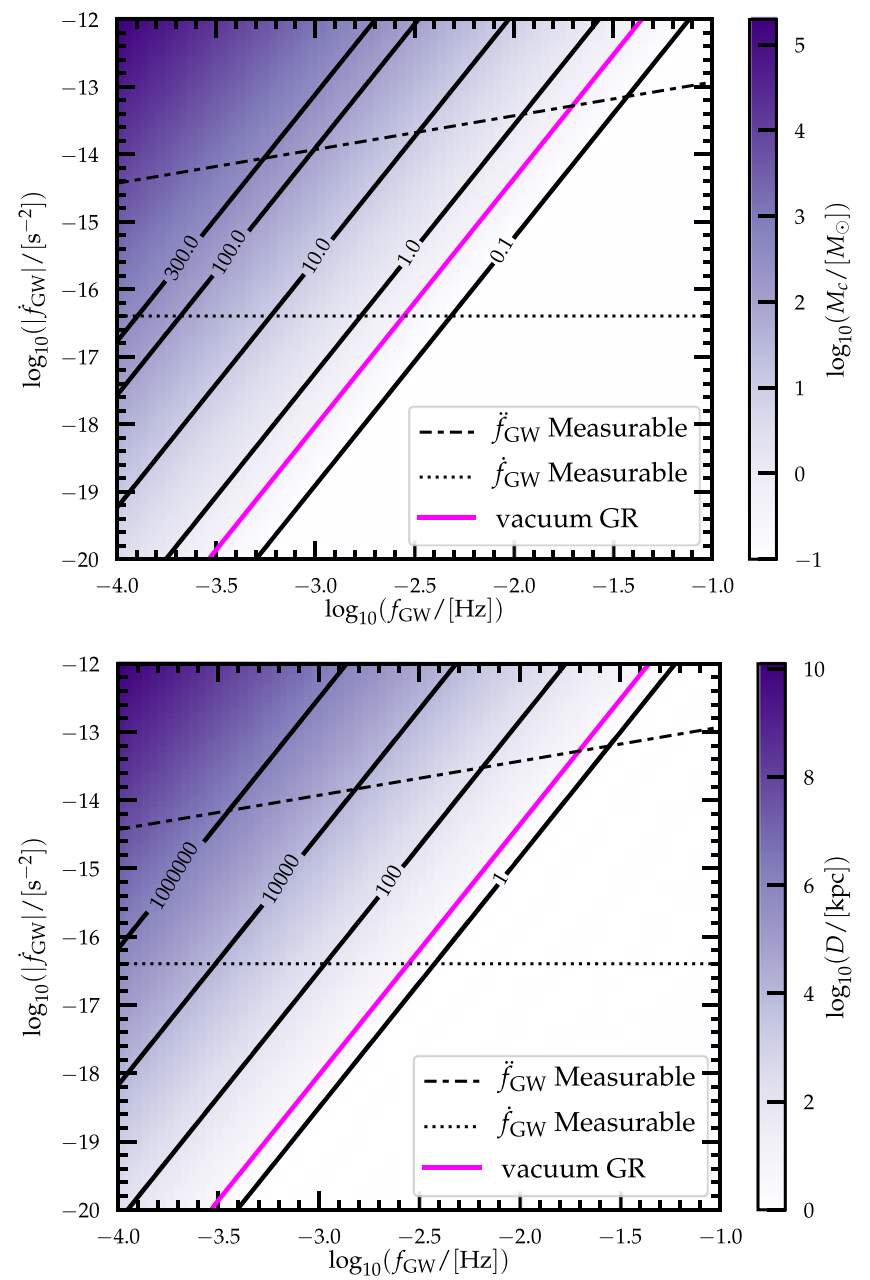

Figure 5. Inferred chirp mass (top) and distance (bottom) for each measured $f_{\mathrm{GW}}$ and $\dot{f}_{\mathrm{GW}}$ for a system with $M_{\text {core }}=0.5 M_{\odot}, M_{\mathrm{WD}}=0.3 M_{\odot}$ at $3 \mathrm{kpc}$. Similar to Figure 3, we use a dashed-dotted line to show the border above which $\ddot{f}_{\mathrm{GW}}$ can be measured and the nature of the binary can be determined through the braking index measurement (see Section 5.1). Below that line there is a possible stealth bias, which these two plots quantify. Above the dotted line, frequency derivatives are likely detectable after five years of observation. Systems below the dotted line would be observed as stationary sources. The magenta line corresponds to the correct value of the chirp mass and distance, namely the GR relation between $f_{\mathrm{GW}}$ and $\dot{f}_{\mathrm{GW}}$ for these masses. Hence the bias vanishes.

2. The (incorrectly) inferred chirp mass may be high enough that the system is consistent with a BBH. In this case, one would expect to continue to see the signal evolve and eventually observe the merger in the LIGO band. Failure to observe the final merger or a signal that is different from a BBH merger signal in vacuum GR would indicate that the binary may have been misidentified.

3. The simultaneous measurement of an apparent high chirp mass and a low distance might indicate the presence of a BBH close to Earth, potentially even in the Galaxy for certain combinations of $f_{\mathrm{GW}}$ and $\dot{f}_{\mathrm{GW}}$. Such a system might be inconsistent with independent information about the BBH inspiral and merger rates obtained from groundbased detectors. This possibility is further discussed in Section 5.2.

If these outcomes fail, the possibility of a stealth bias on the system parameters remains present; it may be impossible to conclusively identify the true nature of the given binary.

\subsection{Measuring the Braking Index}

Stellar binaries evolving purely via GW radiation have GW frequencies that evolve in time as $\dot{f}_{\mathrm{GW}} \propto f_{\mathrm{GW}}^{11 / 3}$. In general, given some source evolving as $\dot{f} \propto f^{n}$, a measurement of the braking index $n$ given by

$$
n=\frac{\ddot{f f}}{\dot{f}^{2}},
$$

is possible if the second time derivative $\ddot{f}$ is measurable. We emphasize the analogy with the pulsar braking index, where $f$ is the pulsar spin frequency rather than the frequency of GWs radiated by the CE binary.

If LISA can successfully measure $f_{\mathrm{GW}}, \dot{f}_{\mathrm{GW}}$, and $\ddot{f}_{\mathrm{GW}}$ for a given GW source, inference on the source's braking index offers a means of identifying the physical processes driving its evolution. In particular, we can seek to verify if $n$ is consistent with $n_{\mathrm{GW}}=\frac{11}{3}$, as expected for pure gravitational radiation in vacuum, or inconsistent with $n_{\mathrm{GW}}$, possibly indicating a binary in the midst of $\mathrm{CE}$ or subject to other environmental influences (e.g., Fedrow et al. 2017).

While LISA is expected to measure $\dot{f}_{\mathrm{GW}}$ for a considerable number of binaries, $\ddot{f}_{\mathrm{GW}}$ is likely measurable only in a much smaller subset of cases. Although determining when $\dot{f}_{\mathrm{GW}}$ and $\ddot{f}_{\mathrm{GW}}$ are measurable requires expensive simulations (Seto 2002; Littenberg et al. 2020), we can make an order-of-magnitude estimate by considering how LISA's frequency resolution scales with time. As LISA accumulates more data, its frequency resolution scales with the observing time $T$ as $\Delta f_{\mathrm{GW}} \approx 1 / T$. Accordingly, we assume that $\dot{f}_{\mathrm{GW}}$ and $\ddot{f}_{\mathrm{GW}}$ are measurable when they cause a binary's frequency to change enough such that it moves from one frequency bin to another over the course of LISA's lifetime. Then, lower limits on the minimum detectable $\dot{f}_{\mathrm{GW}}$ and $\ddot{f}_{\mathrm{GW}}$ are

$$
\begin{gathered}
\dot{f}_{\mathrm{GW}, \min } T=\Delta f_{\mathrm{GW}} \quad \Rightarrow \quad \dot{f}_{\mathrm{GW}, \text { min }} \approx \frac{1}{T^{2}}, \\
\frac{1}{2} \ddot{f}_{\mathrm{GW}, \text { min }} T^{2}=\Delta f_{\mathrm{GW}} \quad \Rightarrow \quad \ddot{f}_{\mathrm{GW}, \text { min }} \approx \frac{2}{T^{3}} .
\end{gathered}
$$

We refer the readers to Takahashi \& Seto (2002) and Robson et al. (2018) for more accurate estimates accounting for the SNR dependence.

On the $\dot{f}_{\mathrm{GW}}-f_{\mathrm{GW}}$ plane, the first condition is a straight line depicted by the black dotted horizontal line in Figures 3 and 5 . We can also identify the region of this plane in which $\ddot{f}_{\mathrm{GW}}$ is measurable for a system evolving in vacuum GR. Using the definition of braking index (Equation (3)) to express $\dot{f}_{\mathrm{GW}}$ as a function of $f_{\mathrm{GW}}$ and $\ddot{f}_{\mathrm{GW}}$, a binary evolving purely via $\mathrm{GW}$ emission with a minimum detectable $\ddot{f}_{\mathrm{GW}}$ (Equation (5)) has a frequency derivative

$$
\dot{f}_{\mathrm{GW}}^{2}=\frac{3}{11} \ddot{f}_{\mathrm{GW}, \min } f_{\mathrm{GW}} .
$$

This relation is plotted in Figures 3 and 5 with the black dasheddotted line. Consider a system above this line. Such a system should have a measurable $\ddot{f}_{\mathrm{GW}}$ (and hence a measurable braking index) if it evolves via GW emission. Therefore, if this system's $\ddot{f}_{\mathrm{GW}}$ or $n$ is not measured, or is measured to be different than the GR prediction, then a non-vacuum or non-GR nature of the binary can be inferred. Next, we consider systems below the line defined 
by Equation (6). For such systems, GW evolution alone cannot lead to a detectable $\ddot{f}_{\mathrm{GW}}$. If $\ddot{f}_{\mathrm{GW}}$ is nevertheless measured, one would infer that the binary does not evolve solely under vacuum GR effects. If $\ddot{f}_{\mathrm{GW}}$ is not measured, then it is not possible to determine the nature of the binary.

\subsection{Distinction from Other Sources Based on Rates}

One potential way to infer the nature of sources that cannot be unambiguously identified using only their GW signature is through their rate of occurrence. For example, if the rate of $\mathrm{CE}$ detections in LISA is higher than the uncertainty in the rate of $\mathrm{BBH}$ mergers, one can statistically filter out the overabundance of $\mathrm{BBH}$ detections in LISA when comparing to the $\mathrm{BBH}$ merger rate from ground-based detectors.

As of LIGO/Virgo O3a, the rate of BBH mergers $\left(29.9_{-8.6}^{+14.9}\right.$ $\mathrm{Gpc}^{-3} \mathrm{yr}^{-1}$, Abbott et al. 2020a, 2020b) is relatively poorly constrained due to the small sample size of $\mathrm{BBH}$ events and the dependence on the assumed $\mathrm{BH}$ mass distribution. However, when LISA will become operational (around the year 2035, AmaroSeoane et al. 2017) the BBH catalog size from ground-based detectors will have increased to approximately $10^{4.5}-10^{5} \mathrm{BBH}$ detections per year (e.g., Baibhav et al. 2019). This means that the constraints on the BBH merger rate will have improved drastically.

The LISA mission will result in an independent measurement of the rate of $\mathrm{BBH}$ mergers, which will be based on the $\mathrm{GW}$ signals from wide (inspiraling) $\mathrm{BBH}$ systems. If the $\mathrm{BBH}$ merger rate as inferred from LISA is significantly higher than the, by then, well constrained $\mathrm{BBH}$ merger rate from groundbased detectors, one could argue that this overabundance could originate from, for example, $\mathrm{CE}$ events.

Moreover, the $\mathrm{BBH}$ merger rate from ground-based detectors will be sufficiently constrained to allow for a split based on event parameters such as $\mathrm{BH}$ mass and spin. This will enable us to constrain, for example, the rate of massive BHs $\left(M_{\mathrm{BH}} \sim\right.$ $\left.40 \mathrm{M}_{\odot}\right)$ in the local Universe. $\mathrm{CE}$ events might appear as more massive BBH events (see Figure 5).

Whether or not the discrepancy between the BBH merger rates inferred from LISA with respect to the ground-based detectors will be significant will depend both on the size of the discrepancy and the uncertainty in the rate measurement from LISA. If we assume that such an overabundance in the rate comes from CE events, then the size of the discrepancy is directly related to the rate of $\mathrm{CE}$ events. Based on our discussion in Section 4 we expect the number of CE events detectable by LISA to lie between 0.1 and 1000. Although this estimate spans several orders of magnitude, we will be able to constrain the upper limit of this estimate (corresponding to long-lasting $\mathrm{CE}$ events with $\left.\Delta t_{\mathrm{CE}} \propto 10^{4} \mathrm{yr}\right)$ given the uncertainty in the stellar mass $\mathrm{BBH}$ merger rate in LISA.

\section{Sky Localization and Electromagnetic Counterparts}

EM transients could provide interesting triggers for $\mathrm{GW}$ searches, and in this case the probability of misinterpreting the signal might be lower. In fact, the self-regulated inspiral phase is expected to follow the dynamical plunge-in phase (step (c) in Figure 1), which might cause optical (e.g., LRNe Soker \& Tylenda 2003; Kulkarni et al. 2007; Ivanova et al. 2013a; Pastorello et al. 2019) and infrared (IR) transients (e.g., SPRITES Kasliwal et al. 2017).

An example of what a possible EM precursor could look like is V1309Sco (Tylenda et al. 2011) and given that the initial parameters of the binary stars are known fairly well, it would be interesting to monitor this object for possible GWs once LISA is operational. Concerning the characterization and discovery of EM precursors, the spectral evolution and late-time observations of V1309Sco suggest that LRNe could produce large amounts of dust and be particularly bright in the infrared (IR; Nicholls et al. 2013; Pejcha et al. 2017; Metzger \& Pejcha 2017; Jencson et al. 2019; Blagorodnova et al. 2020; Iaconi et al. 2020). Therefore, it is conceivable that some of the transients associated with $\mathrm{CE}$ events might only be observable in the IR. The SPitzer InfraRed Intensive Transients Survey (SPIRITS) detected unusual infrared transients with no optical counterparts (Kasliwal et al. 2017). These transients, dubbed eSPecially Red Intermediate-luminosity Transient Events (SPRITEs), are in the infrared luminosity gap between novae and supernovae and could be associated with stellar mergers and $\mathrm{CE}$ events.

Another way to identify possible interesting targets where the $\mathrm{CE}$ event might have been initiated too long ago to detect its beginning is through peculiar circumstellar environments. An example could be TYC 2597-735-1 which was interpreted as a merger product based on its "blue-ring nebula" but still exhibits variability (Hoadley et al. 2020, although its variability is not necessarily related to a still ongoing merger).

Conversely, in the case of a GW detection, the interpretation of the signal might be helped by EM followup. This might be necessary to distinguish a $\mathrm{CE}$ signal from other potential $\mathrm{mHz} \mathrm{GW}$ sources (e.g., AM CVns, sdO+WD binaries). As mentioned before, the most promising GW signal for LISA does not come from the dynamical phase of a $\mathrm{CE}$ associated with the brightest EM transients (e.g., LRNe). Instead, the long-lasting self-regulated inspiral produces a possibly slowly evolving GW source. The LISA sky localization is strongly dependent on the signal and its SNR for a monochromatic source with typical uncertainty ellipses is of order tens of degrees squared (Cutler 1998).

During the self-regulated phase, the CE binary might look like a red giant with the following possible features. The surrounding circumstellar material might be peculiar: a $\mathrm{CE}$ is initiated by an unstable phase of RLOF (step $a$ in Figure 1), which can be nonconservative and spill over in the surroundings of the system (e.g., Pejcha et al. 2017; MacLeod \& Loeb 2020). One-dimensional CE simulations suggest that periodic outbursts of mass ejection during the self-regulated phase are possible (e.g., Clayton et al. 2017). Thus, a CE binary might appear as a red giant with an excess of circumstellar material and peculiar time variability.

\section{Discussion}

\subsection{Comparison to Other Common Envelope Rate Estimates}

Assuming a steady-state stellar population in the Galaxy, the number of $\mathrm{CE}$ binaries viable as $\mathrm{GW}$ sources depends on the initiation rate $R_{\mathrm{CE} \text {,init }}$ and the time duration when the system might be detectable. While presently the latter needs to be estimated from numerical simulations (although, see also Igoshev et al. 2020), the former can be constrained using observations of EM transients associated with CE events.

The most relevant EM transients to constrain the rate of CEs are LRNe, which have been conclusively linked to a CE event via observations of the period decay preceding the outburst event in V1309Sco (Tylenda et al. 2011). Kochanek et al. (2014) estimate the Galactic rate of these events with $V(I)$ band magnitude brighter than $-3(-4)$ to be $\sim 0.5(0.3) \mathrm{yr}^{-1}$, in reasonable agreement with our $R_{\mathrm{CE} \text {,init }}=0.18_{-0.09}^{+0.02} \mathrm{yr}^{-1}$ (see 
Section 4). Their estimate was based on the then available sample of four events in $25 \mathrm{yr}$ and correction for observational biases. Kochanek et al. (2014) also noted the good agreement with population synthesis results obtained with the StarTrack code. More recently, Howitt et al. (2020) used the COMPAS population synthesis code to suggest a Galactic rate of LRNe of $0.2 \mathrm{yr}^{-1}$, which is in agreement with our estimates obtained with COSMIC.

\subsection{Consequences of Non-detection}

Even a non-detection of GWs from a CE event throughout the LISA mission might provide unique and direct constraints on $\mathrm{CE}$ evolution. In this case, regardless of the rate of $\mathrm{CE}$ initiation, the first possibility is that the self-regulated thermal-timescale phase happens at too wide separations, corresponding to $f_{\mathrm{GW}} \lesssim 10^{-4}$ $\mathrm{Hz}$ below the LISA bandpass. For our representative CE binary with $M_{\text {core }}=0.5 M_{\odot}$ and $M_{2}=0.3 M_{\odot}$, this would mean $a_{\text {post-plunge }} \gtrsim 1.5 R_{\odot}$, although from Figure 3 we expect to build up significant SNR only for $a_{\text {post-plunge }} \lesssim 0.5 R_{\odot}$.

Alternatively, direct constraints on the duration in the LISA band $\left(\Delta t_{\mathrm{CE}}\right)$ can be derived with some informed assumptions on the rate of initiation $\left(R_{\mathrm{CE} \text {,init }}\right)$. Considering all CE events, the latter can be estimated with the observed rate of LRNe at $\approx 0.1 \mathrm{yr}^{-1}$ (consistent with our population synthesis calculations; see also Section 7.1). Restricting to CE between two evolved stars with dense cores, our simulations suggest a decrease of about a factor of $\sim 3$ (see Section 4). Therefore, despite the many uncertainties in binary mass transfer stability and CE evolution, both EM transient observations and population synthesis estimates suggest a value of about one CE initiation every decade in the Galaxy. Assuming most of these events will cross the LISA band at some point (see Figure 2) and a mission duration of $T \approx 10 \mathrm{yr}$, a non-detection would be at odds with models for which $\Delta t_{\mathrm{CE}} \gg 1 \mathrm{yr}$, which we predict might result in a significant number of detectable sources. The longer the time $T$ during which a $\mathrm{GW}$ signal might be observed is, the less dependent on uncertainties in $R_{\mathrm{CE}}$, init and more stringent this constraint will be.

\subsection{Further Caveats}

There are several effects that we have not explored in detail in this study which could impact the LISA detectability of a CE event. The dynamical plunge-in could make the orbit inside the $\mathrm{CE}$ eccentric, modifying the GW emission in two ways: make it more "bursty", with stronger emission at each pericenter passage, and make it more "directional." If the rate of CE events is on the optimistic side, LISA can still expect to detect eccentric CE events and will unveil a great deal about the physics of the dynamical plunge-in.

We have also neglected triple stellar systems. Triples can enhance the rate of mass transfer in binaries either because the third star fills its Roche lobe or because of the Kozai-Lidov oscillations inducing high eccentricity in the inner binary (e.g., Toonen et al. 2020). This can in principle enhance the rate of CE and produce events that are qualitatively different in EM and GWs; see, for example, Glanz \& Perets (2021). Hence, our most pessimistic rate calculation may be regarded as a lower limit.

\section{Summary and Conclusions}

Common envelope evolution remains one of the largest uncertainties in stellar physics. Here, we have investigated the possibility of detecting gravitational wave emission from a binary made from the core of the donor star and its companion star inspiraling within a shared common envelope.

Although common envelope evolution is never driven by the loss of energy to gravitational radiation, the binary inside the shared envelope does have a time-dependent mass quadrupole moment and can thus emit gravitational radiation. If detectable, such gravitational radiation offers invaluable insight on the process by passively tracing the motion of the binary.

While the final dynamical phase of a common envelope might emit detectable signals (Ginat et al. 2020), its short duration makes it an extremely rare target. Conversely, we focus on a longer duration phase that might occur in some common envelope events: the self-regulating thermal-timescale phase. Detections of this phase would constrain the stalling radius (from the gravitational wave frequency) and the duration of the stalled phase (from the number of sources detected and/or the signal duration). Even nondetections could put upper limits on the duration or lower limits on the radius at which common envelopes might stall (see Section 7.2).

The gravitational wave frequency range of interest is likely in the $\mathrm{mHz}$ range. This is suggested by the existence of verification white-dwarf binaries for the LISA mission, which are thought to be the outcome of successful common envelope ejections.

Based on rapid population synthesis calculations, we estimate that about one common envelope event is initiated per decade in the Galaxy (see Figure 4), in agreement with previous observational and theoretical determinations (Sections 4 and 7.1). The largest uncertainty in converting this to a number of Galactic sources is the duration of the common envelope, which is predicted to lie between a dynamical ( $\sim$ days) and a thermal (decades to $10^{5} \mathrm{yr}$ ) timescale and is likely to depend on the masses and evolutionary stages of the binary considered. Nevertheless, assuming that some common envelope events go through a selfregulated thermal-timescale phase, it is realistic to expect at least one source within the LISA mission and possibly more than hundreds.

While we remained agnostic on the details of the physics governing the common envelope dynamics, we can bracket the range of possibilities by requiring the common envelope to proceed faster than if driven by pure gravitational wave emission (corresponding to the unrealistic assumption of neglecting the gas drag), and slower than if the gas drag was constant (corresponding to the unrealistic assumption of neglecting the envelope reaction to the inspiraling binary). We found that a system representative of the most common kind of Galactic common envelope-a $0.5 M_{\odot}$ core with a $0.3 M_{\odot}$ companion embedded in a shared envelopemight be (marginally) detectable during the stalled phase within these limits (cf. Figure 3). More specifically, if the separation at which the inspiral stalls is of order of $0.1 R_{\odot}$ and lasts $\gtrsim 5 \mathrm{yr}$, a $\mathrm{SNR}>10$ is possible. The shared envelope is likely of too low density to contribute directly to the gravitational wave signal.

We have further investigated the risk of misinterpreting a future detection of a Galactic common envelope binary if analyzed as a compact binary in vacuum evolving according to general relativity (see Section 5). Although the possibility of misinterpretation exists (cf. Figure 5), there are several ways in which it could be avoided. To break the degeneracies and recognize a signal as the product of a Galactic common envelope, one could couple electromagnetic followup or using electromagnetic observations to attempt directional searches in the gravitational wave data (Section 6), measure deviations from the "braking index" predicted by general relativity (for 
fast evolving signals), or compare the rate of detections with the double compact-object-merger rate from ground-based detectors.

Space-based gravitational wave detectors might improve our understanding the inner dynamics of common envelope evolution by using gravitational waves to probe in a direct way a phenomenon that is not gravitational-wave-driven.

Portions of this study were performed during the LISA Sprint at the Center for Computational Astrophysics of the Flatiron Institute, supported by the Simons Foundation. M.R. thanks S. Justham and Y. F. Jiang for helpful discussions early on during this project and K. Breivik for guidance in using COSMIC and helpful feedback. We thank T. Littenberg for useful discussions on LISA parameter estimation.

Software: COSMIC (Breivik et al. 2020), ipython/ jupyter (Pérez \& Granger 2007), matplotlib (Hunter 2007), NumPy (van der Walt et al. 2011).

\section{Appendix A \\ Calculation of LISA Signal-to-Noise Ratios}

We calculate expected LISA signal-to-noise ratios following the discussion in Robson et al. (2019); our code is available at https://github.com/tcallister/LISA-and-CE-Evolution/. The gravitational wave signal from a quasicircular binary can be generically described via

$$
\begin{aligned}
& h_{+}(t)=A(t) \frac{1+\cos ^{2} \iota}{2} \cos \Psi(t), \\
& h_{\times}(t)=A(t) \cos \iota \sin \Psi(t) .
\end{aligned}
$$

Here, $A(t)$ is the gravitational wave's amplitude, $\iota$ is the inclination of the binary's orbital plane with respect to our line of sight and $\Psi(t)$ is the gravitational wave's phase (the corresponding frequency of gravitational wave emission is $\left.f_{\mathrm{GW}}=\frac{1}{2 \pi} \dot{\Psi}\right)$. In the frequency domain (denoted by a tilde), the resulting signal that is measured by LISA is the linear combination

$$
\tilde{h}\left(f_{\mathrm{GW}}\right)=F_{+}\left(f_{\mathrm{GW}}, \hat{n}\right) \tilde{h}_{+}\left(f_{\mathrm{GW}}\right)+F_{\times}\left(f_{\mathrm{GW}}, \hat{n}\right) \tilde{h}_{\times}\left(f_{\mathrm{GW}}\right),
$$

assuming a signal incident on LISA from direction $\hat{n}$, and where $F_{+}\left(f_{\mathrm{GW}}, \hat{n}\right)$ and $F_{\times}\left(f_{\mathrm{GW}}, \hat{n}\right)$ are the LISA antenna response functions. Note that, unlike ground-based detectors, LISA observes signals whose wavelengths are comparable to the size of the instrument itself and therefore its antenna patterns are strongly frequency-dependent. Also, here we explicitly use $f_{\mathrm{GW}}$ to refer to gravitational wave frequencies in order to minimize confusion with orbital frequencies $f_{\text {orb }}=f_{\mathrm{GW}} / 2$.

The resulting SNR with which this signal is observed in LISA is given by

$$
\mathrm{SNR}^{2}=4 \int_{0}^{\infty} \frac{\left|h\left(f_{\mathrm{GW}}\right)\right|^{2}}{S_{n}\left(f_{\mathrm{GW}}\right)} d f_{\mathrm{GW}},
$$

$S_{n}\left(f_{\mathrm{GW}}\right)$ is the LISA strain sensitivity curve; we adopt the analytic expression in Equation (1) of Robson et al. (2019). As we do not know in advance, the sky location or orientation of possible CE sources, Equation (A3) can be averaged over all sky positions and binary inclination angles, yielding an expected signal-to-noise ratio

$$
\left\langle\mathrm{SNR}^{2}\right\rangle=\frac{16}{5} \int_{0}^{\infty} \frac{\left|\tilde{A}\left(f_{\mathrm{GW}}\right)\right|^{2}}{S_{n}\left(f_{\mathrm{GW}}\right)} d f_{\mathrm{GW}} .
$$

We use Equation (A4) to obtain the results shown with the colors in Figure 3.

To compute the Fourier-domain amplitudes $\tilde{A}\left(f_{\mathrm{GW}}\right)$, we first note that the time-domain amplitude of a quasi-circular binary with chirp mass $\mathcal{M}_{c}$, at distance $D$, and gravitational wave frequency $f_{\mathrm{GW}}$ is (Maggiore 2008)

$$
A(t)=\frac{4}{D}\left(\frac{G \mathcal{M}_{c}}{c^{2}}\right)^{5 / 3}\left(\frac{\pi f_{\mathrm{GW}}}{c}\right)^{2 / 3}
$$

The corresponding Fourier-domain amplitude is (Buonanno et al. 2009)

$$
\tilde{A}\left(f_{\mathrm{GW}}\right)=\frac{A\left(t\left(f_{\mathrm{GW}}\right)\right)}{\sqrt{\dot{f}_{\mathrm{GW}}\left(t\left(f_{\mathrm{GW}}\right)\right)}},
$$

where $\dot{f}_{\mathrm{GW}}$ is the time derivative of the gravitational wave frequency and we now regard time as a function of $f_{\mathrm{GW}}$.

\section{Appendix B \\ Calculation of Gas Drag Neglecting the Envelope Reaction}

Any massive object moving linearly through gas with a constant density interacts with its own gravitational wake and loses momentum via dynamical friction. An object of mass $M$ and radius $R$ traveling with velocity $v$ through gas of uniform density $\rho$ and sound speed $c_{s}$ generates an enhanced density tail. This wake is confined to the Mach cone at supersonic speeds $\left(\mathcal{M}=v / c_{s} \gg 1\right)$ and at sub-sonic speeds the overdense wake lies within a sphere of radius $c_{s} t$ a distance $v t$ behind the mass. If the wake is low amplitude, linear perturbation theory yields a drag force of the form $F_{\mathrm{DF}}=-C 4 \pi(G M)^{2} \rho / v^{2}$ (Ostriker 1999) where $C$ is a term that approaches $C \rightarrow \mathcal{M}^{3} / 3$ as $\mathcal{M} \ll 1$ and $C \rightarrow \ln (v t / R)$ as $\mathcal{M} \gg 1$. For a massive object on a circular orbit, the path of the wake bends and is therefore asymmetric, leading to both azimuthal and radial components of drag. The azimuthal component dominates and is generally well approximated by $F_{\mathrm{DF}}$ (Kim \& Kim 2007). Where the perturber is more massive, the wake is larger amplitude and the nonlinear regime modifies $F_{\mathrm{DF}}$ by an additional factor $(\eta / 2)^{-0.45}$ where $\eta$ is a nonlinear factor given by $\eta=A /\left(\mathcal{M}^{2}-1\right)$ and $A=G M / c_{s}^{2} R_{s}$ with $R_{s}$ a characteristic softening scale (Kim \& Kim 2009). Further modifications to the Kim \& Kim (2007) picture of drag can occur when the embedded mass is a binary and the binary orbit or its orbit around its own center of mass become supersonic (Sánchez-Salcedo \& Chametla 2014).

To calculate the solid orange line in Figure 3, we assumed $\rho=10^{-6} \mathrm{~g} \mathrm{~cm}^{-3}$, roughly corresponding to the average envelope density for a red supergiant and a temperature $T=9 \times 10^{5} \mathrm{~K}$, corresponding to the temperature somewhere inside the envelope. To obtain a (conservative) upper limit on the gas drag force, we consider these parameters constant throughout the inspiral, that is we neglect the reaction of the envelope to the injection of energy and angular momentum by the binary spiraling-in. With these parameters, we calculated $F_{\mathrm{DF}}$ and assumed it to be the only source of energy loss and inspiral. 


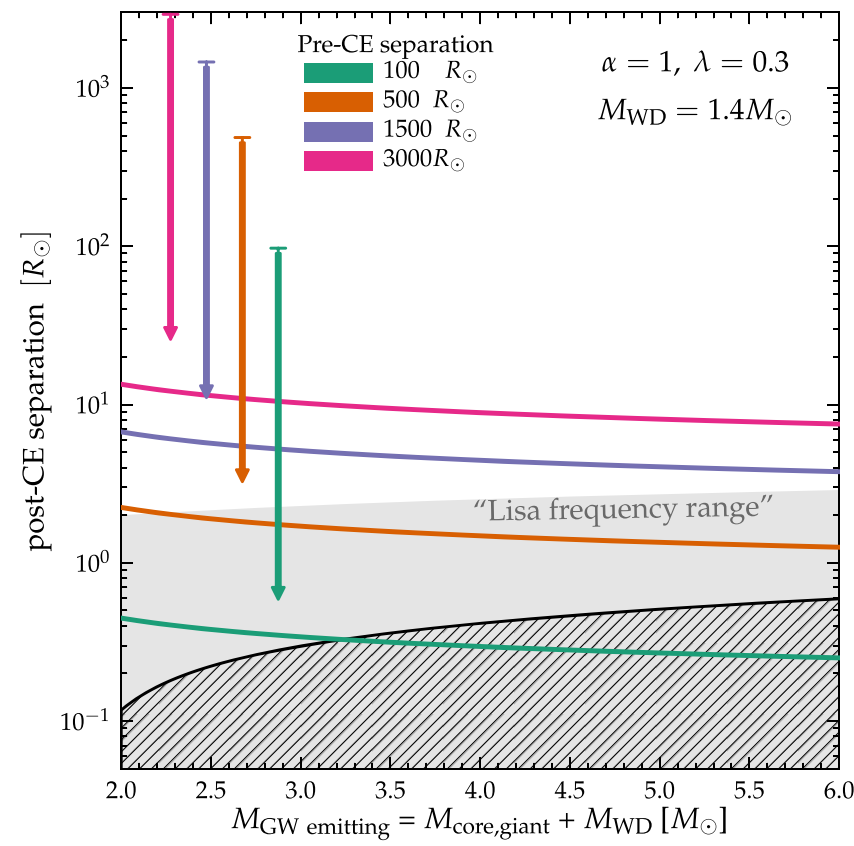

Figure 6. Same as Figure 2 but assuming a compact companion of $1.4 \mathrm{M}_{\odot}$ representing a Chandrasekhar-mass WD or a neutron star.

\section{Appendix C \\ Post-common-envelope Separation with More Massive Companion}

The orbital energy, which in the $\alpha \lambda$ formalism is used to (tentatively) eject the $\mathrm{CE}$, depends on the masses of the stars. Figure 6 shows the predicted post-CE separation as a function of the GW-emitting mass assuming a more massive $1.4 M_{\odot}$ companion (cf. $0.3 M_{\odot}$ in Figure 2). All other parameters are unchanged $(\alpha=1, \lambda=0.3)$.

\section{ORCID iDs}

M. Renzo (1D https://orcid.org/0000-0002-6718-9472

T. Callister (iD https://orcid.org/0000-0001-9892-177X

K. Chatziioannou (D) https://orcid.org/0000-0002-5833-413X

L. A. C. van Son (i) https://orcid.org/0000-0001-5484-4987

C. M. F. Mingarelli (i) https://orcid.org/0000-0002-4307-1322

M. Cantiello (i) https://orcid.org/0000-0002-8171-8596

K. E. S. Ford (1) https://orcid.org/0000-0002-5956-851X

B. McKernan (iD https://orcid.org/0000-0002-9726-0508

G. Ashton (1) https://orcid.org/0000-0001-7288-2231

\section{References}

Aasi, J., Abbott, B. P., Abbott, R., et al. 2015, CQGra, 32, 074001 Abbott, R., Abbott, T. D., Abraham, S., et al. 2020a, arXiv:2010.14527 Abbott, R., Abbott, T. D., Abraham, S., et al. 2020b, arXiv:2010.14533 Acernese, F., Agathos, M., Agatsuma, K., et al. 2015, CQGra, 32, 024001 Amaro-Seoane, P., Audley, H., Babak, S., et al. 2017, arXiv:1702.00786 Baibhav, V., Berti, E., Gerosa, D., et al. 2019, PhRvD, 100, 064060 Baker, J., Bellovary, J., Bender, P. L., et al. 2019, arXiv:1907.06482 Belczynski, K., Holz, D. E., Bulik, T., \& O’Shaughnessy, R. 2016, Natur, 534,512

Blagorodnova, N., Karambelkar, V., Adams, S. M., et al. 2020, MNRAS, 496, 5503

Breivik, K., Coughlin, S., Zevin, M., et al. 2020, ApJ, 898, 71

Broekgaarden, F. S., Berger, E., Neijssel, C. J., et al. 2021, arXiv:2103.02608 Buonanno, A., Iyer, B. R., Ochsner, E., Pan, Y., \& Sathyaprakash, B. S. 2009, PhRvD, 80, 084043
Chamandy, L., Blackman, E. G., Frank, A., Carroll-Nellenback, J., \& Tu, Y. 2020, MNRAS, 495, 4028

Chattopadhyay, D., Stevenson, S., Hurley, J. R., Bailes, M., \& Broekgaarden, F. 2020, arXiv:2011.13503

Chen, W.-C., Liu, D.-D., \& Wang, B. 2020a, arXiv:2008.05143

Chen, X., Xuan, Z.-Y., \& Peng, P. 2020b, ApJ, 896, 171

Claeys, J. S. W., Pols, O. R., Izzard, R. G., Vink, J., \& Verbunt, F. W. M. 2014, A\&A, 563, A83

Clayton, M., Podsiadlowski, P., Ivanova, N., \& Justham, S. 2017, MNRAS, 470, 1788

Cruz-Osorio, A., \& Rezzolla, L. 2020, ApJ, 894, 147

Cutler, C. 1998, PhRvD, 57, 7089

Dan, M., Rosswog, S., Guillochon, J., \& Ramirez-Ruiz, E. 2011, ApJ, 737, 89

Darwin, G. H. 1879, Obs, 3, 79

De Marco, O., Passy, J.-C., Moe, M., et al. 2011, MNRAS, 411, 2277

de Mink, S. E., Langer, N., Izzard, R. G., Sana, H., \& de Koter, A. 2013, ApJ, 764, 166

Di Carlo, U. N., Mapelli, M., Bouffanais, Y., et al. 2020, MNRAS, 497, 1043

Dominik, M., Belczynski, K., Fryer, C., et al. 2012, ApJ, 759, 52

Duchêne, G., \& Kraus, A. 2013, ARA\&A, 51, 269

Evans, F. A., Renzo, M., \& Rossi, E. M. 2020, MNRAS, 497, 5344

Fedrow, J. M., Ott, C. D., Sperhake, U., et al. 2017, PhRvL, 119, 171103

Fragos, T., Andrews, J. J., Ramirez-Ruiz, E., et al. 2019, ApJL, 883, L45

Ginat, Y. B., Glanz, H., Perets, H. B., Grishin, E., \& Desjacques, V. 2020, MNRAS, 493, 4861

Glanz, H., \& Perets, H. B. 2018, MNRAS, 478, L12

Glanz, H., \& Perets, H. B. 2021, MNRAS, 500, 1921

Glebbeek, E., Gaburov, E., de Mink, S. E., Pols, O. R., \& Portegies Zwart, S. F. 2009, A\&A, 497, 255

Götberg, Y., Korol, V., Lamberts, A., et al. 2020, ApJ, 904, 56

Han, Z., Podsiadlowski, P., Maxted, P. F. L., \& Marsh, T. R. 2003, MNRAS, 341,669

Hoadley, K., Martin, D. C., Metzger, B. D., et al. 2020, Natur, 587, 387

Howitt, G., Stevenson, S., Vigna-Gómez, A. R., et al. 2020, MNRAS, 492, 3229

Huang, S.-J., Hu, Y.-M., Korol, V., et al. 2020, PhRvD, 102, 063021

Hunter, J. D. 2007, CSE, 9, 90

Hurley, J. R., Tout, C. A., \& Pols, O. R. 2002, MNRAS, 329, 897

Iaconi, R., Maeda, K., Nozawa, T., De Marco, O., \& Reichardt, T. 2020, MNRAS, 497, 3166

Igoshev, A. P., Perets, H. B., \& Michaely, E. 2020, MNRAS, 494, 1448

Ivanova, N. 2018, ApJL, 858, L24

Ivanova, N., Justham, S., Avendano Nandez, J. L., \& Lombardi, J. C. 2013a, Sci, 339, 433

Ivanova, N., Justham, S., Chen, X., et al. 2013b, A\&ARv, 21, 59

Ivanova, N., Justham, S., \& Ricker, P. 2020, Common Envelope Evolution (Bristol: Institute of Physics Publishing), 2514

Ivanova, N., Podsiadlowski, P., \& Spruit, H. 2002, MNRAS, 334, 819

Jencson, J. E., Kasliwal, M. M., Adams, S. M., et al. 2019, ApJ, 886, 40

Justham, S., Wolf, C., Podsiadlowski, P., \& Han, Z. 2009, A\&A, 493, 1081

Kasliwal, M. M., Bally, J., Masci, F., et al. 2017, ApJ, 839, 88

Kim, H., \& Kim, W.-T. 2007, ApJ, 665, 432

Kim, H., \& Kim, W.-T. 2009, ApJ, 703, 1278

Kochanek, C. S., Adams, S. M., \& Belczynski, K. 2014, MNRAS, 443, 1319

Korol, V., Rossi, E. M., Groot, P. J., et al. 2017, MNRAS, 470, 1894

Kroupa, P., Tout, C. A., \& Gilmore, G. 1993, MNRAS, 262, 545

Kruckow, M. U., Tauris, T. M., Langer, N., Kramer, M., \& Izzard, R. G. 2018, MNRAS, 481, 1908

Kulkarni, S. R., Ofek, E. O., Rau, A., et al. 2007, Natur, 447, 458

Kupfer, T., Bauer, E. B., Marsh, T. R., et al. 2020, ApJ, 891, 45

Law-Smith, J. A. P., Everson, R. W., Ramirez-Ruiz, E., et al. 2020, arXiv:2011.06630

Littenberg, T., Cornish, N., Lackeos, K., \& Robson, T. 2020, Phys. Rev. D, 101,123021

Liu, W.-M., Jiang, L., \& Chen, W.-C. 2021, arXiv:2101.09850

MacLeod, M., \& Loeb, A. 2020, ApJ, 895, 29

MacLeod, M., Macias, P., Ramirez-Ruiz, E., et al. 2017, ApJ, 835, 282

MacLeod, M., Ostriker, E. C., \& Stone, J. M. 2018, ApJ, 868, 136

MacLeod, M., \& Ramirez-Ruiz, E. 2014, ApJL, 798, L19

Maggiore, M. 2008, Gravitational Waves: Volume 1: Theory and Experiments (Oxford: Oxford Univ. Press), https://books.google.com/books? id=AqVpQgAACAAJ

Metzger, B. D., \& Pejcha, O. 2017, MNRAS, 471, 3200

Meyer, F., \& Meyer-Hofmeister, E. 1979, A\&A, 78, 167

Michaely, E., \& Perets, H. B. 2019, MNRAS, 484, 4711

Moe, M., \& Di Stefano, R. 2017, ApJS, 230, 15 
Nandez, J. L. A., Ivanova, N., \& Lombardi, J. C. J. 2015, MNRAS, 450, L39 Nariai, K., \& Sugimoto, D. 1976, PASJ, 28, 593

Nelemans, G., Verbunt, F., Yungelson, L. R., \& Portegies Zwart, S. F. 2000 A\&A, 360, 1011

Neunteufel, P. 2020, A\&A, 641, A52

Nicholls, C. P., Melis, C., Soszynski, I., et al. 2013, MNRAS, 431, L33

Ohlmann, S. T., Röpke, F. K., Pakmor, R., Springel, V., \& Müller, E. 2016, MNRAS, 462, L121

Ostriker, E. C. 1999, ApJ, 513, 252

Paczyński, B. 1976, in IAU Symp. 73: Structure and Evolution of Close Binary Systems, ed. P. Eggleton, S. Mitton, \& J. Whelan (Cambridge: Cambridge Univ. Press), 75

Pastorello, A., Mason, E., Taubenberger, S., et al. 2019, A\&A, 630, A75

Pastorello, A., Valerin, G., Fraser, M., et al. 2020, arXiv:2011.10590

Pavlovskii, K., Ivanova, N., Belczynski, K., \& Van, K. X. 2017, MNRAS, 465, 2092

Pejcha, O., Metzger, B. D., Tyles, J. G., \& Tomida, K. 2017, ApJ, 850, 59

Pérez, F., \& Granger, B. E. 2007, CSE, 9, 21

Podsiadlowski, P. 2001, in ASP Conf. Ser., 229, Common-Envelope Evolution and Stellar Mergers (San Francisco, CA: ASP), 239

Podsiadlowski, P., Rappaport, S., \& Pfahl, E. D. 2002, ApJ, 565, 1107

Pols, O. R., Schröder, K.-P., Hurley, J. R., Tout, C., \& Eggleton, P. 1998, MNRAS, 298, 525

Renzo, M., Cantiello, M., Metzger, B. D., \& Jiang, Y. F. 2020, ApJL, 904, L13

Renzo, M., Zapartas, E., de Mink, S. E., et al. 2019, A\&A, 624, A66

Ricker, P. M., \& Taam, R. E. 2008, ApJL, 672, L41

Robson, T., Cornish, N. J., \& Liu, C. 2019, CQGra, 36, 105011
Robson, T., Cornish, N. J., Tamanini, N., \& Toonen, S. 2018, PhRvD, 98, 064012

Sánchez-Salcedo, F. J., \& Chametla, R. O. 2014, ApJ, 794, 167

Sand, C., Ohlmann, S. T., Schneider, F. R. N., Pakmor, R., \& Röpke, F. K. 2020, A\&A, 644, A60

Sberna, L., Toubiana, A., \& Miller, M. C. 2020, arXiv:2010.05974

Seto, N. 2002, MNRAS, 333, 469

Soker, N., \& Tylenda, R. 2003, ApJL, 582, L105

Somiya, K. 2012, CQGra, 29, 124007

Stroeer, A., \& Vecchio, A. 2006, CQGra, 23, S809

Takahashi, R., \& Seto, N. 2002, ApJ, 575, 1030

Tamanini, N., \& Danielski, C. 2019, NatAs, 3, 858

Tauris, T. M., Kramer, M., Freire, P. C. C., et al. 2017, ApJ, 846, 170

Temmink, K. D., Toonen, S., Zapartas, E., Justham, S., \& Gänsicke, B. T. 2020, A\&A, 636, A31

Thorne, K. S., \& Żytkow, A. N. 1977, ApJ, 212, 832

Toonen, S., Portegies Zwart, S., Hamers, A. S., \& Bandopadhyay, D. 2020 A\&A, 640, A16

Tylenda, R., Hajduk, M., Kamiński, T., et al. 2011, A\&A, 528, A114

van der Walt, S., Colbert, S. C., \& Varoquaux, G. 2011, CSE, 13, 22

Vigna-Gómez, A., MacLeod, M., Neijssel, C. J., et al. 2020, PASA, 37, e038

Voss, R., \& Tauris, T. M. 2003, MNRAS, 342, 1169

Webbink, R. F. 1984, ApJ, 277, 355

Wilson, E. C., \& Nordhaus, J. 2020, MNRAS, 497, 1895

Wong, K. W. K., Breivik, K., Kremer, K., \& Callister, T. 2021, PhRvD, 103, 083021

Zapartas, E., de Mink, S. E., Izzard, R. G., et al. 2017, A\&A, 601, A29

Zevin, M., Bavera, S. S., Berry, C. P. L., et al. 2020, arXiv:2011.10057 